\title{
Unusual charge density wave transition and absence of magnetic ordering in $\mathrm{Er}_{2} \mathrm{Ir}_{3} \mathrm{Si}_{5}$
}

\author{
Sitaram Ramakrishnan, ${ }^{1}$ Andreas Schönleber $\odot,{ }^{1}$ Toms Rekis $\odot,{ }^{1}$ Natalija van Well $\odot,{ }^{1, *}$ Leila Noohinejad, ${ }^{1, \dagger}$ \\ Sander van Smaalen ${ }^{0},{ }^{1, \ddagger}$ Martin Tolkiehn, ${ }^{2}$ Carsten Paulmann, ${ }^{3}$ Biplab Bag, ${ }^{4}$ Arumugam Thamizhavel, ${ }^{4}$ \\ Dilip Pal, ${ }^{5}$ and Srinivasan Ramakrishnan ${ }^{4, \S}$ \\ ${ }^{1}$ Laboratory of Crystallography, University of Bayreuth, 95447 Bayreuth, Germany \\ ${ }^{2}$ P24, PETRA III, DESY, 22607 Hamburg, Germany \\ ${ }^{3}$ Mineralogisch-Petrographisches Institut, Universität Hamburg, 20146 Hamburg, Germany \\ ${ }^{4}$ Department of Condensed Matter Physics and Materials Science, Tata Institute of Fundamental Research, Mumbai 400005, India \\ ${ }^{5}$ Department of Physics, Indian Institute of Technology, Guwahati 781039, India
}

(Received 1 December 2019; accepted 27 January 2020; published 10 February 2020)

\begin{abstract}
The first-order charge density wave (CDW) phase transition of $\mathrm{Er}_{2} \mathrm{Ir}_{3} \mathrm{Si}_{5}$ is characterized by a crystal structure analysis, and electrical resistivity, magnetic susceptibility and specific heat measurements. The incommensurate CDW is accompanied by a strong lattice distortion, from which it is shown that the CDW resides on zigzag chains of iridium atoms. The CDW transition affects the magnitude of the local magnetic moments on $\mathrm{Er}^{3+}$, implying strong coupling between CDW and magnetism. This could account for the observation that magnetic order is suppressed down to at least $0.1 \mathrm{~K}$ in the high-quality single crystal presently studied. Any disorder in the crystallinity, as in ceramic material, broadens and suppresses the CDW transition, while magnetic order appears at $2.1 \mathrm{~K}$.
\end{abstract}

DOI: 10.1103/PhysRevB.101.060101

The study of competing phase transitions is one of the frontier areas of condensed matter physics [1]. A large number of investigations have been made to understand the competition between and coexistence of local-moment magnetism, superconductivity, giant magnetoresistance, and charge density wave (CDW) ordering in both inorganic and organic compounds. Many of these materials possess one-dimensional (1D) or two-dimensional (2D) crystal structures [2-5]. The low dimensionality of these compounds is central to the exotic ground states exhibited by these compounds.

In contrast to these low-dimensional compounds, CDWs seem to occur in several compounds with complex threedimensional (3D) structures. Crystal structures of these compounds do not show obvious $1 \mathrm{D}$ or $2 \mathrm{D}$ features, while their electrical conductivity is only weakly anisotropic. These materials include the element uranium [6], the rare-earth $(R)$ containing compounds $R \mathrm{Te}_{3}$ [7], $R \mathrm{Te}_{2}$ [8], $R \mathrm{NiC}_{2}$ [1,9-11], $R_{5} \mathrm{Ir}_{4} \mathrm{Si}_{10}$ [12], and $R_{2} \mathrm{Ir}_{3} \mathrm{Si}_{5}$ [13-15], as well as $\mathrm{CuV}_{2} \mathrm{~S}_{4}$ [16]. Usually one observes a coexistence of the CDW with magnetism or superconductivity in the above-mentioned systems. However, in recently discovered $R \mathrm{NiC}_{2}$ [11,17], the CDW competes with magnetism and in the extreme case ferromagnetic order destroys the CDW in $\mathrm{SmNiC}_{2}[9,18,19]$, suggesting strong coupling of the rare-earth spins to the CDW in this series. Even in the series $R \mathrm{NiC}_{2}$, where $R$ is a magnetic

\footnotetext{
*Present address: Department for Earth- and Environmental Sciences, Crystallography Section, Ludwig-Maximilians-University Munich, 80333 Munich, Germany.

†Present address: P24, PETRA III, DESY, 22607 Hamburg, Germany.

${ }^{\ddagger}$ smash@uni-bayreuth.de

§ramky@tifr.res.in
}

rare-earth element, the normal state magnetic susceptibility is unaffected by the CDW transition.

Nonmagnetic $\mathrm{Lu}_{2} \mathrm{Ir}_{3} \mathrm{Si}_{5}$ becomes superconducting below $T_{\mathrm{sc}}=3.5 \mathrm{~K}$, and it undergoes a first-order CDW transition between 150 and $200 \mathrm{~K}$, which is governed by strong electronphonon coupling [13]. Kuo et al. [14] established these transitions in their polycrystalline sample at temperatures of 5.5 and $110-160 \mathrm{~K}$, respectively. A lowering of the transition temperature of the presumed CDW transition with a concomitant increase of the superconducting transition is strong evidence for the involvement of the Fermi surface in the CDW transition and thus for the CDW character of this transition [14]. Furthermore, sample-dependent transition temperatures indicate a sensitive dependence of the transition on intrinsic disorder or lattice defects, which is again in agreement with the CDW character of this transition. The second characteristic of the CDW state is the presence of a periodic lattice distortion (PLD). Lee et al. [15] have discovered incommensurate satellite reflections in the electron diffraction of $\mathrm{Lu}_{2} \mathrm{Ir}_{3} \mathrm{Si}_{5}$ measured at temperatures below $T_{\mathrm{CDW}}$, which indicate an incommensurate PLD/CDW with modulation wave vector $\mathbf{q}=\delta(\overline{1} 21)$ and $\delta=0.23-0.25$.

A signature of possible CDW transitions was not found for polycrystalline samples of the other compounds $R_{2} \mathrm{Ir}_{3} \mathrm{Si}_{5}$ [20], except for a broad anomaly in the electrical resistivity of $\mathrm{Er}_{2} \mathrm{Ir}_{3} \mathrm{Si}_{5}$ [21]. Unlike the polycrystalline material, a single crystal of $\mathrm{Er}_{2} \mathrm{Ir}_{3} \mathrm{Si}_{5}$ showed very sharp anomalies with a clear hysteresis in the temperature dependencies of the electrical resistivity and magnetic susceptibility [22].

Here, we show that the incommensurate CDW is accompanied by a structural transition, resulting in a lowering of the crystal symmetry from orthorhombic to triclinic. Evidence for the involvement of the Ir atoms in CDW formation is obtained from a detailed description of the incommensurate crystal 
structure of the CDW state. We show that magnetic ordering is suppressed in single crystals, while a previous report has found $T_{N}=2.2 \mathrm{~K}$ for polycrystalline $\mathrm{Er}_{2} \mathrm{Ir}_{3} \mathrm{Si}_{5}$, where the CDW transition is suppressed [20]. Cooling through the CDW transitions leads to an increased magnetic susceptibility, while $\mathrm{Er}_{2} \mathrm{Ir}_{3} \mathrm{Si}_{5}$ remains paramagnetic, making it a unique CDW system where there is strong coupling of the Er spins to CDW ordering.

Single crystals of $\mathrm{Er}_{2} \mathrm{Ir}_{3} \mathrm{Si}_{5}$ were grown in a tetra-arc furnace from a stoichiometric mixture of the elements, employing a modified Czochralski technique. The purity of the reactants was $99.99 \%$ for Er and Ir, and $99.999 \%$ for silicon. The 2-3-5 stoichiometry was established by energy-dispersive $\mathrm{x}$-ray analysis (EDAX). X-ray diffraction confirmed the expected lattice parameters.

A small part of the as-grown single crystal was crushed, and a piece of $0.05 \times 0.08 \times 0.06 \mathrm{~mm}^{3}$ was selected for single-crystal x-ray diffraction. Diffraction experiments were performed at Beamline P24 of PETRA-III extension at DESY in Hamburg, employing radiation of a wavelength of $0.49994 \AA$ A The temperature of the specimen was controlled by a CRYOCOOL open-flow helium gas cryostat. Complete data sets of intensities of Bragg reflections were measured at temperatures of 20, 75, and $130 \mathrm{~K}$ (CDW phase) and 150 and 200 K (normal phase) [23] (see also Refs. [24-26] therein).

The crystallographic orientation of the as-grown single crystal was determined by the backscattering Laue diffraction method, employing the ORIENT EXPRESS software [27]. The observed sharp Bragg reflections provided independent evidence for the single-crystalline nature of the sample. Small bars of required orientations were cut off the oriented crystal by a wire saw and used for transport and magnetization measurements.

The electrical resistivity was measured by the four-point method along the c direction of an oriented single crystal. Electrical contacts were applied by gluing gold wires $(40 \mu \mathrm{m}$ diameter) with silver paste to the crystal surface. The electrical resistivity was measured with a custom-built setup based on a LR-700 (Linear Research, USA) AC bridge, operating with a current of $5 \mathrm{~mA}$ at a frequency of $16 \mathrm{~Hz}$. Resistivity data were measured for temperatures between 1.5 and $300 \mathrm{~K}$ with a cooling and heating rate of $1 \mathrm{~K} / \mathrm{min}$ (Fig. 1). A very sharp anomaly is observed, that starts at $T=150 \mathrm{~K}$ and reaches a maximum at $149 \mathrm{~K}$, while cooling. Upon heating, the anomaly starts at $T=166 \mathrm{~K}$ and reaches a minimum value at $168 \mathrm{~K}$. It signifies the opening of a gap in the electronic density of states over a major fraction of the Fermi surface, in agreement with a charge density wave (CDW) transition. The hysteresis of $17.0 \mathrm{~K}$ and the sharpness of the transition indicate a firstorder CDW transition. A similarly sharp transition has been observed for single crystals of $\mathrm{Lu}_{2} \mathrm{Ir}_{3} \mathrm{Si}_{5}$ [28], as well as for the commensurate $\mathrm{CDW}$ in $\mathrm{Lu}_{5} \mathrm{Ir}_{4} \mathrm{Si}_{10}$ [29], suggestive of a similar mechanism for CDW formation in these compounds. A much more gradual transition, but still with hysteresis, has been observed for ceramic samples of $\mathrm{Lu}_{2} \mathrm{Ir}_{3} \mathrm{Si}_{5}$ and $\mathrm{Er}_{2} \mathrm{Ir}_{3} \mathrm{Si}_{5}[13,14,20]$. The sensitivity of the CDW transition for crystal defects furthermore follows from the electrical resistivity measured on single crystals of $\mathrm{Er}_{2} \mathrm{Ir}_{3} \mathrm{Si}_{5}$ of lesser quality, which indicated a broadened transition (see preliminary reports by one of us $[22,30])$.

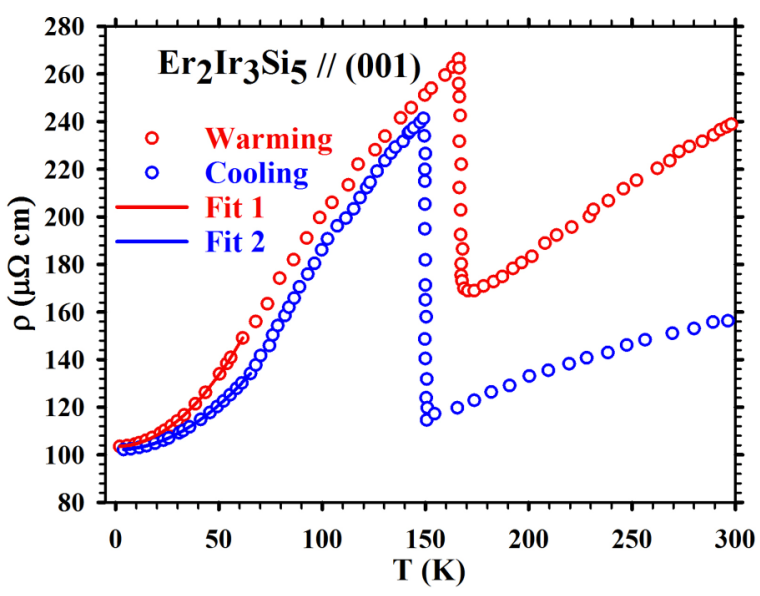

FIG. 1. Temperature dependence of the electrical resistivity $\rho$ along the $\mathbf{c}$ axis of $\mathrm{Er}_{2} \mathrm{Ir}_{3} \mathrm{Si}_{5}$, as measured between 1.5 and $300 \mathrm{~K}$, during cooling (blue circles) and heating (red circles) of the sample. The solid lines are fits to the low-temperature data (see text).

Another important feature is the different values of the electrical resistivity obtained on cooling and subsequent heating of the sample. Similar behavior has been observed for ceramic material of $\mathrm{Lu}_{2} \mathrm{Ir}_{3} \mathrm{Si}_{5}[13,14]$. It has been explained by variations of pinning of the CDW. Alternatively, it is possible that the large lattice distortions across the CDW transition are responsible for the formation of microcracks, a reduction of sizes of mosaic blocks, and an increase in texture, which, in turn, would cause an increase of the electrical resistance at each thermal cycle. A mechanism by microcracks is supported by the observation that the transition temperatures and hysteresis are the same in each thermal cycle. An increase of pinning centers would lead to a lowering of the CDW transition temperature, which is not observed.

It is interesting to note that the electrical resistivity exhibits a $T^{2}$ dependence below $T<50 \mathrm{~K}\left[\rho(T)=\rho_{0}+A T^{2}\right.$; fit 1 and fit 2 in Fig. 1], i.e., up to much higher temperatures than $\sim 10 \mathrm{~K}$ for a Fermi liquid, implying dominant contributions from short-range magnetic fluctuations in the absence of magnetic ordering.

The specific heat $\left(C_{p}\right)$ was measured by the thermal relaxation method, using a physical property measuring system (PPMS, Quantum Design, USA). Data obtained during heating of a single crystal of $11 \mathrm{mg}$ from 2 to $250 \mathrm{~K}$ exhibit a sharp peak at the temperature of the CDW transition at 163.8(1) K (Fig. 2). We are unable to find a peak in $C_{p}(T)$ while cooling the crystal. Similar behavior was noted earlier for a crystal of $\mathrm{Lu}_{2} \mathrm{Ir}_{3} \mathrm{Si}_{5}$ [28]. This feature could be the result of the specific method of measurement employed in the PPMS instrument. In a second experiment, differential scanning calorimetry (DSC) was measured from 120 to $200 \mathrm{~K}$ on the same single crystal of $\mathrm{Er}_{2} \mathrm{Ir}_{3} \mathrm{Si}_{5}$. DSC data show sharp peaks in both heating and cooling runs, that appear at similar temperatures as is found for the electrical resistivity, and thus confirm the first-order phase transition (Fig. 3). The transition is even sharper than what was found for $\mathrm{Lu}_{2} \mathrm{Ir}_{3} \mathrm{Si}_{5}$ [28]). This feature might be related to the high quality of the present single crystal (see discussion above).

Below $10 \mathrm{~K}, C_{p}(T)$ could be fitted to $\gamma T+\beta T^{3}$, resulting in a high value of $\gamma=0.545 \mathrm{~J} /\left(\mathrm{mol} \mathrm{K}^{2}\right)$ and a Debye 


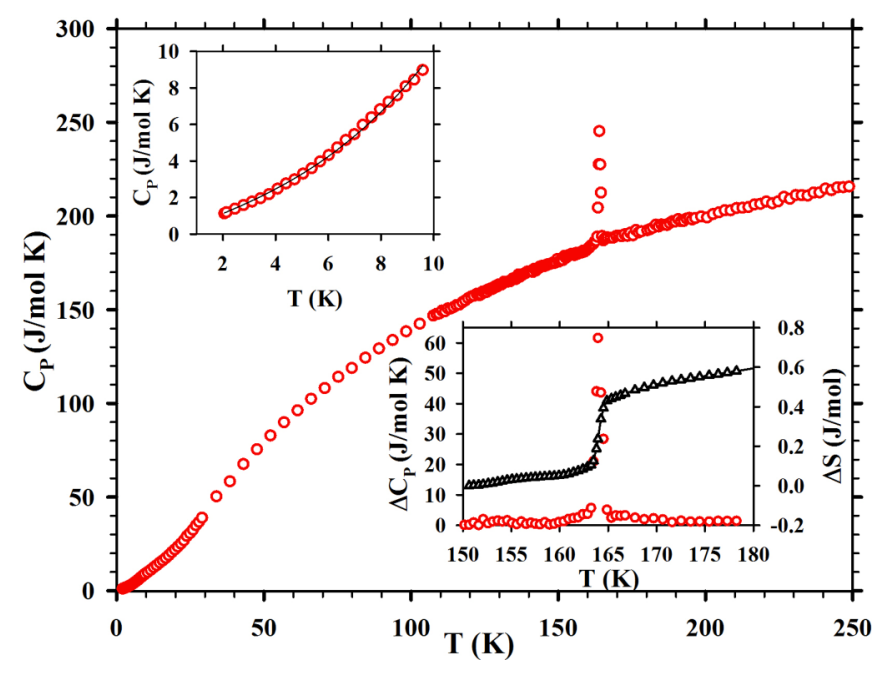

FIG. 2. The specific heat $\left(C_{p}\right)$ of a single crystal of $\mathrm{Er}_{2} \mathrm{Ir}_{3} \mathrm{Si}_{5}$ upon heating from 2 to $250 \mathrm{~K}$. The upper inset shows the specific heat data below $10 \mathrm{~K}$; the solid line is a fit to the data points (see text). The lower inset provides an expanded view around the phase transition of $\Delta C_{p}$ (see text) and the increase of entropy $\Delta S$ due to the phase transition.

temperature of $\theta_{D}=350 \mathrm{~K}$ (upper panel of Fig. 2). Along with the $T^{2}$ dependence of the electrical resistivity, the high value of $\gamma$ is in agreement with short-range antiferromagnetic correlations of the magnetic moments of $\mathrm{Er}^{3+}$.

The lattice contribution to the specific heat was determined from a fit to the data far away from the transition. Subtraction of the lattice contribution resulted in $\Delta C_{p}(T)$ (Fig. 2). The change of entropy at the transition $\Delta S(T)$ has been determined by integration of $\Delta C_{p}(T) / T$ over temperature (lower inset in Fig. 2). The resulting value of $\Delta S=0.06 R$ is comparable to transition entropies obtained for conventional CDW systems, but it is seven to ten times smaller than for $R_{5} \mathrm{Ir}_{4} \mathrm{Si}_{10}$ [12] and $\mathrm{Lu}_{2} \mathrm{Ir}_{3} \mathrm{Si}_{5}$ [28]). On the other hand, the specific heat anomaly indicates a much sharper transition

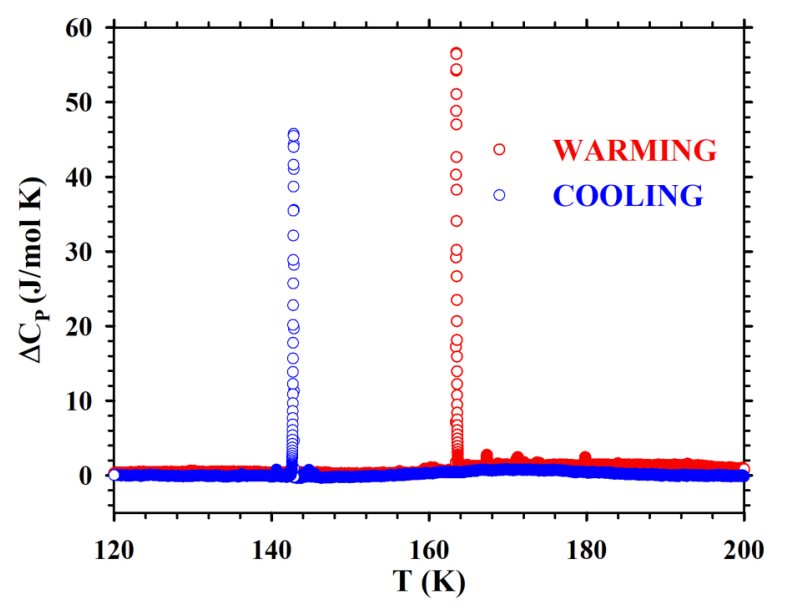

FIG. 3. Temperature dependence of $\Delta C_{p}$ of $\mathrm{Er}_{2} \mathrm{Ir}_{3} \mathrm{Si}_{5}$, obtained by subtracting a smooth background from the measured DSC data. A peak is found at $T=142.8(1) \mathrm{K}$ upon cooling (blue circles), and at $T=163.6(1) \mathrm{K}$ upon heating (red circles).

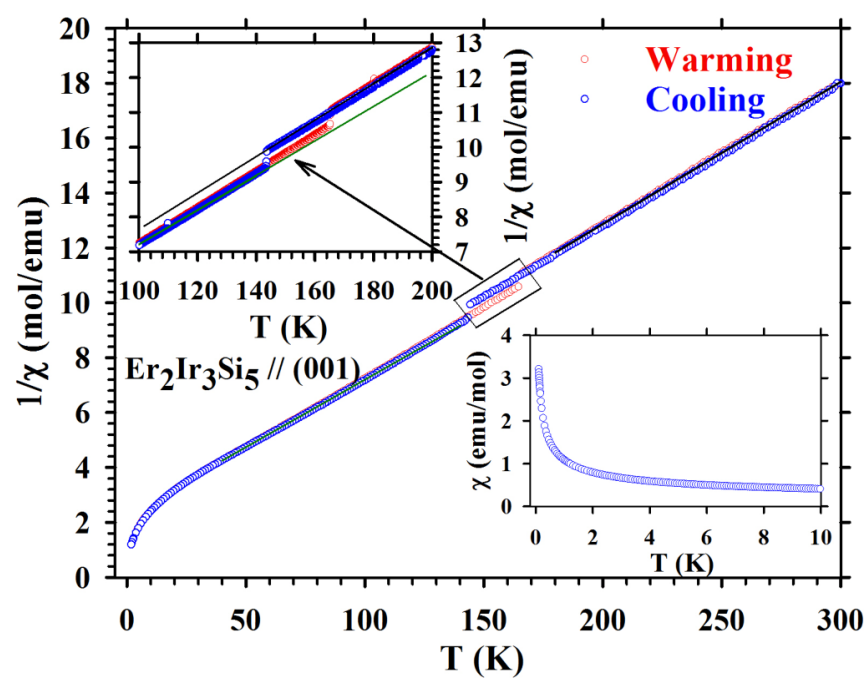

FIG. 4. Temperature dependence of the magnetic susceptibility $\chi$ along $\mathbf{c}$ of a single crystal of $\mathrm{Er}_{2} \mathrm{Ir}_{3} \mathrm{Si}_{5}$. The main panel shows $\frac{1}{\chi}$ for cooling (blue circles) and heating (red circles) of the sample between 2 and $300 \mathrm{~K}$. The upper inset gives an enlarged view of the hysteresis observed around the CDW transition, with solid lines describing the results of a Curie-Weiss fit to data for $180-300 \mathrm{~K}$ and a Curie-Weiss fit to data for $40-140 \mathrm{~K}$ (see text). The lower inset gives $\chi(T)$ at low temperatures, and clearly indicates the absence of magnetic ordering down $0.1 \mathrm{~K}$.

for single crystals of $\mathrm{Er}_{2} \mathrm{Ir}_{3} \mathrm{Si}_{5}$ than for conventional CDW systems, which is in agreement with the first-order character of the transition. Furthermore, the specific heat anomaly with $\Delta C_{p}(\max )=61.65(5) \mathrm{J} /(\mathrm{mol} \mathrm{K})$ at $T=163.6(1) \mathrm{K}$ is much larger than for conventional CDW systems, such as $\mathrm{K}_{0.3} \mathrm{MoO}_{3}$ $\left[\Delta C_{p}(\max )=8 \mathrm{~J} /(\mathrm{mol} \mathrm{K}) ; \Delta S=0.18 R\right][31-33]$ and $\mathrm{NbSe}_{3}$ $\left[\Delta C_{p}(\max ) \cong 9 \mathrm{~J} /(\operatorname{mol~K}) ; \Delta S=0.08 R\right]$ [34]. Large values of $\Delta C_{p}(\max )$ have also been found for $\mathrm{Lu}_{5} \mathrm{Ir}_{4} \mathrm{Si}_{10}$ [29] and $\mathrm{Lu}_{2} \mathrm{Ir}_{3} \mathrm{Si}_{5}$ [28]). It is in agreement with substantial lattice distortions accompanying the CDW transition (see below).

The temperature-dependent magnetic susceptibility $\chi(T)$ was measured with a commercial superconducting quantum interference device (SQUID) magnetometer (MPMS 7, Quantum Design, USA) along the c direction of a single crystal during cooling and during heating of the sample between 2 and $300 \mathrm{~K}$, and employing a magnetic field of $0.1 \mathrm{mT}$ (Fig. 4). In a second experiment, $\chi(T)$ was measured with a custom-built setup for temperatures between 0.1 and $10 \mathrm{~K}$, again employing a magnetic field of $0.1 \mathrm{mT}$ (lower inset in Fig. 4). The data show the absence of magnetic order down to $0.1 \mathrm{~K}$.

The most interesting feature of the temperature-dependent magnetic susceptibility is the sudden increase of $\chi(T)$ at 144.0(1) $\mathrm{K}$ upon cooling through the CDW transition. The observed effect cannot be explained by a change of Pauli susceptibility at the transition, because the estimated magnitude of the Pauli susceptibility is at least two orders of magnitude smaller than the observed jump of the susceptibility. Furthermore, one would expect a smaller Pauli susceptibility in the CDW phase, whereas we observe an increase of the susceptibility when cooling through the transition. The same transition is found at 164(1) K upon heating. The observed 
hysteresis is in good agreement with the hysteresis found in the electrical resistivity and DSC measurements. A CurieWeiss fit to the data at 180-300 K results in a Curie constant $C=19.29(1) \mathrm{emu} / \mathrm{mol} \mathrm{K}$ and an antiferromagnetic Weiss temperature of $\theta=-48.2(1) \mathrm{K}$. A Curie-Weiss fit to the low-temperature paramagnetic regime $40-140 \mathrm{~K}$ results in $C=20.34(1) \mathrm{emu} / \mathrm{mol} \mathrm{K}$ and $\theta=-46.4(1) \mathrm{K}$. The different Curie constants correspond to different effective magnetic moments on $\mathrm{Er}^{3+}$ of $8.78 \mu_{\mathrm{B}}$ and $9.02 \mu_{\mathrm{B}}$, respectively.

$\mathrm{Er}_{2} \mathrm{Ir}_{3} \mathrm{Si}_{5}$ is an exceptional case in showing an effect of the CDW transition on the magnetic susceptibility. Usually, compounds containing magnetic rare-earth elements do not show any anomaly in the paramagnetic susceptibility at the high-temperature CDW transitions. For example, $\mathrm{Er}_{5} \operatorname{Ir}_{4} \mathrm{Si}_{10}$ is paramagnetic for at least 3-300 $\mathrm{K}$ without any anomalies in the magnetic susceptibility at its CDW transition [35]. For this compound, the Curie-Weiss fit resulted in a magnetic moment on $\mathrm{Er}^{3+}$ of $9.7 \mu_{\mathrm{B}}$, which represents the theoretical value of free $\mathrm{Er}^{3+}$ ions [35]. Furthermore, the interaction between magnetic moments appears to be much weaker in $\mathrm{Er}_{5} \mathrm{Ir}_{4} \mathrm{Si}_{10}$ (fitted value $\theta=-3.0 \mathrm{~K}$ ) than in $\mathrm{Er}_{2} \mathrm{Ir}_{3} \mathrm{Si}_{5}$. The coexistence of antiferromagnetic order $\left(T_{N}=2.8 \mathrm{~K}\right)$ and $\mathrm{CDW}$ in $\mathrm{Er}_{5} \mathrm{Ir}_{4} \mathrm{Si}_{10}$ might be related to the presence of weakly coupled independent $4 f$ electrons of $\mathrm{Er}^{3+}$ ions [36], while for $\mathrm{Er}_{2} \mathrm{Ir}_{3} \mathrm{Si}_{5}$ the reduced magnitude of the magnetic moments and strong coupling suggest participation of the $4 f$ electrons in the conduction bands. It is not clear at this moment, whether the small but distinct change of $\mathrm{Er}^{3+}$ moment $\left(8.78 \mu_{\mathrm{B}}\right.$ vs $\left.9.02 \mu_{\mathrm{B}}\right)$ across the CDW transition in a single crystal of $\mathrm{Er}_{2} \mathrm{Ir}_{3} \mathrm{Si}_{5}$ could be responsible for absence of magnetic ordering of $\mathrm{Er}^{3+}$ moments in the crystal down to $0.1 \mathrm{~K}$.

Single-crystal x-ray diffraction (SXRD) indicates a strong monoclinic lattice distortion with $\beta=91.695(2)^{\circ}$ at $T=$ $75 \mathrm{~K}$ in the CDW phase [Table S3 in the Supplemental Material (SM) [23]]. The lattice distortion is accompanied by superlattice reflections that can be indexed by incommensurate modulation wave vectors [Eq. (S1) in SM [23]]

$$
\begin{aligned}
& \mathbf{q}^{1}=[0.2495(2), 0.4973(1), 0.2483(1)], \\
& \mathbf{q}^{3}=[0.2495(2),-0.4973(1), 0.2483(1)] .
\end{aligned}
$$

Related $\mathbf{q}$ vectors describe satellite reflections from the other domains. The modulation wave vectors resemble those observed in $\mathrm{ED}$ on $\mathrm{Lu}_{2} \mathrm{Ir}_{3} \mathrm{Si}_{5}$, while the monoclinic lattice distortion was not noticed in ED, because it cannot be visualized in the [101] zone diffraction patterns studied in Ref. [15].

Based on the observed lattice distortion, two monoclinic structure models can be devised for the CDW phase. The first model preserves the $I$ center of the lattice and it has a 2D modulation, involving both modulation wave vectors [Eq. (1)]. The second model assumes the second component of each wave vector to be exactly $\pm 1 / 2$; then $\mathbf{q}^{1}-\mathbf{q}^{3}=(0,1,0)$ and the $I$ center is lost. The CDW superstructure would become a mixture of a commensurate supercell (loss of the $I$ center corresponds to doubling of the unit cell) and a 1D incommensurate modulation according to $\mathbf{q}^{1}$. However, neither of these two models is compatible with the diffraction data (Table S5 in SM [23]). Instead, the CDW crystal structure is triclinic, preserves the $I$ center, and has a $1 \mathrm{D}$ incommensurate modulation according to the superspace group $I \overline{1}(\alpha \beta \gamma) 0$ [No. 2.1.1.1 with

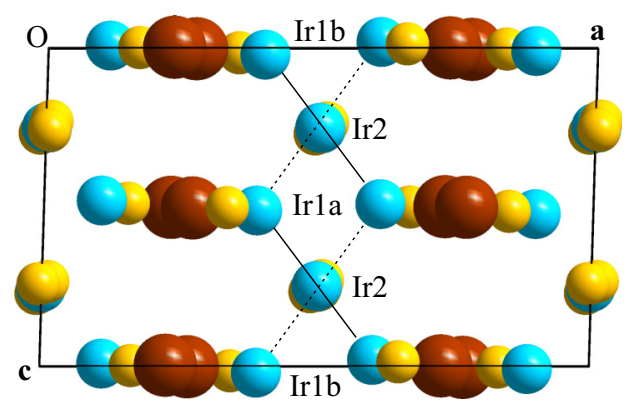

FIG. 5. The crystal structure of $\mathrm{Er}_{2} \mathrm{Ir}_{3} \mathrm{Si}_{5}$ at $75 \mathrm{~K}$ projected onto the $(\mathbf{a}, \mathbf{c})$ plane. Large red spheres correspond to Er atoms; blue spheres of intermediate size correspond to Ir atoms; small yellow spheres are $\mathrm{Si}$ atoms. Ir $1 \mathrm{a}-\mathrm{Ir} 1 \mathrm{~b}$ atoms are at a basic-structure distance of 3.763(6) $\AA$ for the dashed lines and of 3.390(6) $\AA$ for the solid lines.

standard setting $P \overline{1}\left(\alpha^{\prime} \beta^{\prime} \gamma^{\prime}\right) 0$ [37]] with modulation wave vector $\mathbf{q}^{1}=[0.2495(2), 0.4973(1), 0.2483(1)]$ and lattice parameters $a=9.8498(3), b=11.4863(3), c=5.7268(2) \AA$, and $\alpha=90.079(1), \beta=91.695(2)$, and $\gamma=90.051(1)^{\circ}$ at $T=75 \mathrm{~K}$ (Table S3 in SM [23]). The large monoclinic lattice distortion accompanying the incommensurate $\mathrm{CDW}$ transition makes $\mathrm{Er}_{2} \mathrm{Ir}_{3} \mathrm{Si}_{5}$ a unique CDW system without a counterpart in the literature.

Both aspects, the lattice distortion and the 1D incommensurate modulation, appear to be important for the formation of the CDW. The orthorhombic Ibam crystal structure, stable above $T_{\mathrm{CDW}}$, has six crystallographically independent atoms, Er1, Ir1, Ir2, Si1, Si2, and Si3 (Table S7 in SM [23]). The triclinic basic structure has ten independent atoms in the unit cell (Table S8). In particular, Ir1 atoms form a regular zigzag chain along $\mathbf{c}$ in Ibam with distance $d[\operatorname{Ir} 1-\operatorname{Ir} 1]=3.666(1) \AA$. In $I \overline{1}$, Ir1 splits into Ir1a and Ir1b sites, with the zigzag chain described as (Ir1a, $\operatorname{Ir} 1 \mathrm{~b})_{\infty}$ (Fig. 5). The lattice distortion is responsible for the formation of a dimerized zigzag chain with alternating distances $d[\operatorname{Ir} 1 \mathrm{a}-\operatorname{Ir} 1 \mathrm{~b}]=3.390(6)$ and 3.763(6) $\AA$. It then is the shorter distance of these two which is mostly affected by the incommensurate modulation (Fig. 6). The result is a strong modulation along the zigzag chain of Ir1, with one distance nearly constant at $3.76 \AA$ and the other distance varying between 3.1 and $3.8 \AA$. These features

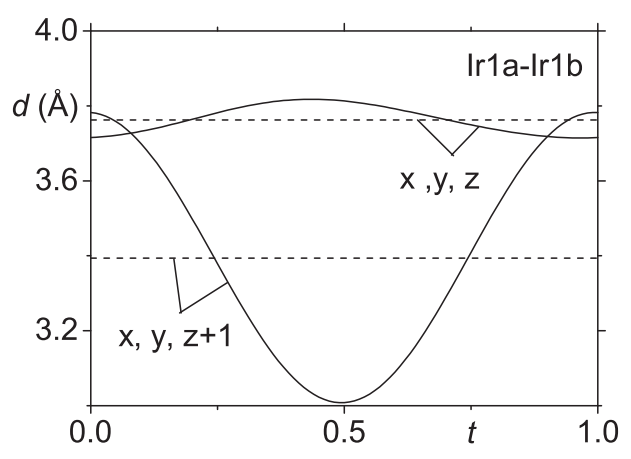

FIG. 6. $t$-Plot of the interatomic distances between atoms Ir1a and $\operatorname{Ir} 1 \mathrm{~b}(x, y, z)$ and between $\operatorname{Ir} 1 \mathrm{a}$ and $\operatorname{Ir} 1 \mathrm{~b}$ at $(x, y, z+1)$ for the crystal structure at $T=75 \mathrm{~K}$. Dashed lines give the distances in the basic structure, with values of 3.763(6) and 3.390(6) $\AA$. 
strongly suggest that the zigzag chains of Ir1 atoms along c are responsible for the CDW. Er1 atoms also are arranged in zigzag chains along c. However, distances Er1-Er1 are around $4 \AA$ and they exhibit a much smaller modulation than the short Ir1a-Ir1b distance. Direct participation of Er1 in the CDW thus is not expected.

In conclusion, we have described in full detail the incommensurately modulated crystal structure of the CDW phase of $\mathrm{Er}_{2} \mathrm{Ir}_{3} \mathrm{Si}_{5}$. As we have shown, the incommensurate CDW is accompanied by a strong lattice distortion, both of which are important for the modulation of interatomic distances on zigzag chains of iridium atoms along $\mathbf{c}$. These features are in agreement with the CDW being supported by these zigzag chains. A presumed role of Er atoms could be excluded. A combination of a lattice distortion and incommensurate modulation was previously found for $\mathrm{Er}_{5} \mathrm{Ir}_{4} \mathrm{Si}_{10}$ [38]. This suggests similarities between the mechanisms for CDW formation in these two compounds. Nevertheless, the change of entropy at the CDW transition in $\mathrm{Er}_{2} \mathrm{Ir}_{3} \mathrm{Si}_{5}$ is similar to typical CDW systems, while it is much smaller than in the 3D CDW system $\mathrm{Er}_{5} \mathrm{Ir}_{4} \mathrm{Si}_{10}$ (Fig. 2 and Ref. [12]).

The large lattice distortion explains the sluggish character and large hysteresis of the transition, as they are apparent in the temperature dependencies of the electrical resistivity, DSC, and magnetic susceptibility. A unique feature of compounds $R_{2} \mathrm{Ir}_{3} \mathrm{Si}_{5}$ is the extreme sensitivity of the phase transitions on crystalline order. The present single crystals of high perfection undergo a CDW transition, while magnetic order is suppressed down to at least $0.1 \mathrm{~K}$. Previous experiments on polycrystalline material have observed a broader CDW transition, while magnetic order appears below $T_{N}=$ $2.1 \mathrm{~K}[20,21]$. This is qualitatively different from $\mathrm{Lu}_{2} \mathrm{Ir}_{3} \mathrm{Si}_{5}$, for which superconductivity is weakly affected by the crystal perfection [28]. It demonstrates an intimate competition between CDW and magnetic order, presumably both interactions employing the same part of the Fermi surface, which is not the case in $\mathrm{Er}_{5} \mathrm{Ir}_{4} \mathrm{Si}_{10}$, where Er spins are not affected by the CDW ordering. Accordingly, $\mathrm{Er}_{2} \mathrm{Ir}_{3} \mathrm{Si}_{5}$ provides a different test bed for studies towards the competition between CDW and magnetism of the Er spins and the influence of disorder on these phase transitions.

We thank D. D. Buddikot for his help in measuring the heat capacity. L. Eiter is thanked for his assistance with preliminary x-ray diffraction experiments. X-ray diffraction experiments with synchrotron radiation were performed at station EH2 of beamline P24 of PETRA-III at DESY in Hamburg, Germany. This research has been funded by the Deutsche Forschungsgemeinschaft (DFG, German Research Foundation) Grant No. 265092781, and by the Alexander von Humboldt Foundation within their research-group linkage program.
[1] M. Roman, J. Strychalska-Nowak, T. Klimczuk, and K. K. Kolincio, Phys. Rev. B 97, 041103(R) (2018).

[2] G. Grüner, Charge Density Waves in Solids (Addison-Wesley, Reading, MA, 1994).

[3] P. Rogl, in Handbook of Physics and Chemistry of Rare Earths, edited by K. A. J. Gschneidner and L. Eyring (North-Holland, Amsterdam, 1984), Vol. 7, pp. 1-264.

[4] J. Leciejewicz and A. Szytula, in Handbook of Physics and Chemistry of Rare Earths, edited by K. A. J. Gschneidner and L. Eyring (North-Holland, Amsterdam, 1989), Vol. 12, pp. 133-211.

[5] H. F. Braun, J. Less Common Met. 100, 105 (1984).

[6] J. C. Marmeggi, A. Delapalme, G. H. Lander, C. Vettier, and N. Lehner, Solid State Commun. 43, 577 (1982).

[7] E. DiMasi, M. C. Aronson, J. F. Mansfield, B. Foran, and S. Lee, Phys. Rev. B 52, 14516 (1995).

[8] E. DiMasi, B. Foran, M. C. Aronson, and S. Lee, Phys. Rev. B 54, 13587 (1996).

[9] S. Shimomura, C. Hayashi, G. Asaka, N. Wakabayashi, M. Mizumaki, and H. Onodera, Phys. Rev. Lett. 102, 076404 (2009).

[10] S. Shimomura, C. Hayashi, N. Hanasaki, K. Ohnuma, Y. Kobayashi, H. Nakao, M. Mizumaki, and H. Onodera, Phys. Rev. B 93, 165108 (2016).

[11] K. K. Kolincio, M. Roman, M. J. Winiarski, J. StrychalskaNowak, and T. Klimczuk, Phys. Rev. B 95, 235156 (2017).

[12] S. Ramakrishnan and S. van Smaalen, Rep. Prog. Phys. 80, 116501 (2017).

[13] Y. Singh, D. Pal, S. Ramakrishnan, A. M. Awasthi, and S. K. Malik, Phys. Rev. B 71, 045109 (2005).
[14] Y. K. Kuo, K. M. Sivakumar, T. H. Su, and C. S. Lue, Phys. Rev. B 74, 045115 (2006).

[15] M. H. Lee, C. H. Chen, M.-W. Chu, C. S. Lue, and Y. K. Kuo, Phys. Rev. B 83, 155121 (2011).

[16] R. M. Fleming, F. J. DiSalvo, R. J. Cava, and J. V. Waszczak, Phys. Rev. B 24, 2850 (1981).

[17] M. Murase, A. Tobo, H. Onodera, Y. Hrano, T. Hosaka, S. Shimomura, and N. Wakabayashi, J. Phys. Soc. Jpn. 73, 2790 (2004).

[18] G.-H. Gweon, J. D. Denlinger, J. A. Clack, J. W. Allen, C. G. Olson, E. DiMasi, M. C. Aronson, B. Foran, and S. Lee, Phys. Rev. Lett. 81, 886 (1998).

[19] A. Wölfel, L. Li, S. Shimomura, H. Onodera, and S. van Smaalen, Phys. Rev. B 82, 054120 (2010).

[20] Y. Singh, D. Pal, and S. Ramakrishnan, Phys. Rev. B 70, 064403 (2004).

[21] R. Padam, A. Thamizhavel, S. Ravi, S. Ramakrishnan, and D. Pal, in Proceedings of the 56th DAE Solid State Physics Symposium 2011, edited by R. Mittal, A. K. Chauhan, and R. Mukhopadhyay, AIP Conf. Proc. No. 1447 (AIP, Melville, NY, 2012), p. 1007

[22] P. C. Lalngilneia, A. Thamizhavel, S. Ramakrishnan, and D. Pal, J. Phys.: Conf. Ser. 592, 012094 (2015).

[23] See Supplemental Material at http://link.aps.org/supplemental/ 10.1103/PhysRevB.101.060101 for details on the diffraction experiments, values of the structural parameters at each temperature, and $t$-plots of interatomic distances.

[24] A. M. M. Schreurs, X. Xian, and L. M. J. Kroon-Batenburg, J. Appl. Crystallogr. 43, 70 (2010).

[25] G. M. Sheldrick, SADABS, version 2008/1 (University of Göttingen, Göttingen, 2008). 
[26] V. Petř́íček, M. Dušek, and L. Palatinus, Z. Kristallogr. - Cryst. Mater. 229, 345 (2014).

[27] J. Laugier and B. Bochu, LMGP-Suite Suite of Programs for the Interpretation of $X$-ray Experiments (ENSP/Laboratoire des Matériaux et du Génie Physique, Saint Martin d'Hères, France, 2004).

[28] N. S. Sangeetha, A. Thamizhavel, C. V. Tomy, S. Basu, A. M. Awasthi, P. Rajak, S. Bhattacharyya, S. Ramakrishnan, and D. Pal, Phys. Rev. B 91, 205131 (2015).

[29] B. Becker, N. G. Patil, S. Ramakrishnan, A. A. Menovsky, G. J. Nieuwenhuys, J. A. Mydosh, M. Kohgi, and K. Iwasa, Phys. Rev. B 59, 7266 (1999).

[30] P. C. Lalngilneia, A. Thamizhavel, S. Ramakrishnan, and D. Pal, in Proceedings of the 58th DAE Solid State Physics Symposium 2013, edited by C. Murli, D. Bhattacharyya, and S. C. Gadkari, AIP Conf. Proc. No. 1591 (AIP, Melville, NY, 2014), p. 113.

[31] J. W. Brill, M. Chung, Y.-K. Kuo, X. Zhan, E. Figueroa, and G. Mozurkewich, Phys. Rev. Lett. 74, 1182 (1995).
[32] R. S. Kwok and S. E. Brown, Phys. Rev. Lett. 63, 895 (1989).

[33] R. S. Kwok, G. Gruner, and S. E. Brown, Phys. Rev. Lett. 65, 365 (1990).

[34] S. Tomić, K. Biljaković, D. Djurek, J. Cooper, P. Monceau, and A. Meerschaut, Solid State Commun. 38, 109 (1981).

[35] F. Galli, R. Feyerherm, R. W. A. Hendrikx, S. Ramakrishnan, G. J. Nieuwenhuys, and J. A. Mydosh, Phys. Rev. B 62, 13840 (2000).

[36] F. Galli, R. Feyerherm, R. W. A. Hendrikx, E. Dudzik, G. J. Nieuwenhuys, S. Ramakrishnan, S. D. Brown, S. van Smaalen, and J. A. Mydosh, J. Phys.: Condens. Matter 14, 5067 (2002).

[37] H. T. Stokes, B. J. Campbell, and S. van Smaalen, Acta Crystallogr., Sect. A 67, 45 (2011).

[38] F. Galli, S. Ramakrishnan, T. Taniguchi, G. J. Nieuwenhuys, J. A. Mydosh, S. Geupel, J. Ludecke, and S. van Smaalen, Phys. Rev. Lett. 85, 158 (2000). 